\title{
Analyzing the neutron scattering angular distribution in a ACE-formatted multigroup cross section library using the maximum entropy method
}

\author{
Shuai-tao ZHU, Xu-bo MA *, Hui TANG, Le YANG, and Bo CAO \\ School of Nuclear Science and Engineering, North China Electric Power University, Beijing 102206, China
}

\begin{abstract}
To describe the angular distribution for a group-to-group neutron scattering event, the coefficients of a truncated Legendre polynomial are usually used in the multigroup cross section. However, the truncated expansion can caused negative values of a probability density function (PDF) in some scattering angles. In this study, the PDF of scattering angles is calculated using the maximum entropy method to avoid the negative probabilities of some scattering angles and the multigroup cross section library in ACE format is generated. The multigroup cross section library is verified by the computation of RBEC-M benchmark using RMC. The differences between two methods generating scattering data, the maximum entropy method and discrete angle method, are also discussed. The calculation results using multigroup cross section library in ACE format are well good agreement with the results of benchmark report. It shows that multigroup cross section library including the scattering angle data computed by the maximum entropy method is corrected and can be used for Monte Carlo calculation.
\end{abstract}

\section{Introduction}

In some cases of criticality safety analysis and transport computation, because geometrical detail rather than cross section detail is the dominant consideration, Monte Carlo methods handling multigroup calculations are preferred over those based on deterministic transport theory. In a number of applications using particle transport codes based on Monte Carlo methods, there are some advantages for calculations of multigroup cross sections compared with calculations of point wise cross sections[1], such as reliabilities of multigroup cross sections used in the deterministic transport codes and the results computed by deterministic transport codes can be verified by multigroup Monte Carlo calculations, the adjoint calculations with multigroup Monte Carlo method can be used in problems where it is more efficient than forward transport calculations, the adjoint importance functions generated by multigroup Monte Carlo calculations can enhance the calculational efficiency in forward multigroup or continuous energy Monte Carlo calculations. However, when the anisotropic scattering is considered, the multigroup treatment probably brings ray effects because of limited discrete directions. Effective use of the Monte Carlo multigroup method requires the availability of appropriate multigroup cross sections. The sampling of scattering angles from the multigroup transfer matrices given by deterministic codes is a difficult problem for multigroup Monte Carlo codes. The scattering angular distribution of the multigroup transport equation is usually expanded by Legendre polynomials and the $\mathrm{L}$ order truncated is made to represent the scattering anisotropy phenomenon. But the truncated expansion can be negative for some scattering angles, leading to the negative scattering source and flux. To deal with this problem, several methods have been proposed. The MORSE code has used the discrete angle representation[2], which can be obtained easily by an analytical procedure and precisely preserves the moments. But it may bring ray effects. To eliminate the ray effects, the equally probable step function representation conserving approximately the required moment has been proposed by Carter and Forest[3]. But it fails in the case of a mixture. The none equally probable step function representation preserving the requirements for a medium with single nuclide or for a mixture has been proposed by $\mathrm{Li}$ Mao and J.C.Nimal[4, 5]. The shortcoming for this representation rests in that the scattering angle data cannot be used in some Monte Carlo codes, such as RMC[6]. To obtain group-to-group scattering angles of mixtures, this paper adopts the maximum entropy method proposed by O.B.Moskalev[7]. RMC (Reactor Monte Carlo Code) can use nuclear data presented in ACE (a compact ENDF) format in both point-wise or group-wise representation. The theory of ACE-formatted multigroup cross section library generation is discussed in Section 1. In Section 2, the definitions of the discrete angle representation and the equally probable step function representation are reviewed. Section 3 and 4 discuss the reliability of multigroup cross section library in ACE format including the scattering angle data computed by the maximum entropy method. The last section provides the conclusions of this work.

*e-mail: maxb@ncepu.edu.cn 


\section{Theory of Cross Section Process}

\subsection{The procedure of generating multigroup cross section library in ACE format}

Firstly, multigroup cross sections of each isotope are produced by NJOY Code based on ENDF/B-VII.1. Then MGGC (MultiGroup Cross section Generation Code) works with multigroup cross section library in MATXS format. MGGC determines the background cross sections using the mixture and geometry information provided by the user and interpolates for the effective cross sections[8]. Different isotopes in the same zone of a fast reactor can be set to different temperatures to meet the special calculation requirements and multigroup macroscopic cross sections in ISOTXS format of homogenized mixtures can be generated by MGGC. Besides, MGGC can also generate microscopic cross sections of each isotope and collapse multigroup cross sections. Finally, the format of the multigroup cross section library is transformed from ISOTXS format to ACE format by MGXSMC code. The procedure to produce the ACE-formatted multigroup cross section (XS) library is shown in figure 1.

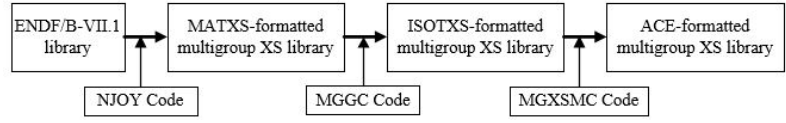

Figure 1. Procedure of generating multigroup XS Library in ACE fromat

\subsection{Resonance self-shielding model}

Resonance self-shielding treatment plays an important role in the procedure of generating multigroup cross section Library, whose essence is to get the problem dependent spectrum used as weighting function to calculate effective self-shielded cross-sections. The resonance cross section of heavy nuclides makes the problem more complicated within a fuel rod. There are many resonance nuclides in the fuel and the resonance peaks of different nuclides overlap each other. When the self-shielded cross sections of one nuclide are calculated, the effect of the overlap on energy spectrum should be taken into account. This is known as the interference effect. The multigroup form of neutron flux can be derived by the Bondarenko method based on the narrow resonance approximation, considering the interference effect. It is written as:

$$
\phi_{l, g}^{i}=\frac{C_{g}}{\left(\sigma_{0, g}^{i}+\sigma_{t, g}^{i}\right)^{l+1}}
$$

where $C_{g}$ is the weight spectrum of the group $g, \sigma_{t, g}^{j}$ and $\sigma_{0, g}^{j}$ are the total microscopic cross section and background cross section of isotope $j, l$ is the order of Legendre series. The calculation of the background cross section, considering the interference effect of each isotope is determined by the Eq. 2:

$$
\sigma_{0, g}^{i}=\frac{1}{N_{i}} \sum_{j \neq i} N_{j} \sigma_{t, g}^{j}\left(\sigma_{t, g}^{i}\right)+\frac{1}{N_{i} \bar{l}}
$$

The first term on the right equation is the appropriate background cross section for an infinite homogeneous mixture. The second term represents the geometry effect, which will also increase the background cross section. Where $N_{i}$ is the number density for isotope $i, \bar{l}$ is the average chord length of medium.

\section{Method for Sampling the Scattering Angle}

The neutron scattering angular distribution from group $g$ to $g$ can be expanded in a series of Legendre polynomials with truncated at $L$ :

$$
f_{L, g \rightarrow g^{\prime}}(\mu)=\frac{1}{2 \pi} \sum_{l=0}^{L} \frac{2 l+1}{2} f_{l, g \rightarrow g^{\prime}} P_{l}(\mu)
$$

where $\mu$ is the cosine of the scattering angle in the laboratory system. Statistical errors in Monte Carlo calculation will be taken place due to negative particle weight in the random walk introduced by the negative distribution.

\subsection{Discrete Angle Representation}

The discrete angle method is based on a discrete distribution which has its first moments identical to those of Eq. 4:

$$
f_{L, g \rightarrow g^{\prime}}(\mu)=\sum_{i=0}^{(L+1) / 2} w_{i} \delta\left(\mu-\mu_{i}\right)
$$

where $w_{i}$ is the probability of $\mu_{i}$. The cosine of the scattering angles and their probabilities can be obtained by the generalized Gaussian quadrature treatment. The discrete directions tend to be located near the peaks of the scattering distribution. The advantage of using this method avoids the negative $f_{L, g \rightarrow g^{\prime}}(\mu)$. But ray effects may occur in a low order Legendre expansion case.

\subsection{Exponential Function Representation}

While approximating an unknown probability distribution, the maximum entropy principle reveals that the best approach to ensure that the approximation satisfies any constraints on the unknown distribution that we are aware of, the distribution should have maximum entropy. For our purpose, the entropy of a probability distribution function of scattering angles is defined as:

$$
S=\int_{-1}^{1} \tilde{g}(\mu) \ln (1 / \tilde{g}(\mu)) d \mu
$$

The $(L+1)$ constraints are expressed as:

$$
\int_{-1}^{1} \tilde{g}(\mu) P_{l}(\mu) d \mu-f_{l}=0,(l=0,1, \ldots, L)
$$

where $P_{l}(\mu)$ is the Legendre polynomial, $f_{l}$ is the coefficient of the Legendre polynomial. The solution of the maximum entropy problem can be written as:

$$
\tilde{g}(\mu)=\int_{-1}^{1} e^{\sum_{l=0}^{L} \lambda_{l} P_{l}(\mu)}
$$


where $\lambda_{l}$ are the Lagrange multiplies that can be obtained by solving $(L+1)$ nonlinear equations. Then the step boundaries of scattering angles can be generated by Eq. 7 . Avoiding ray effects is the main advantage of the Equally Probable Step Function Representation.

\section{Verification Benchmark}

For verification of the proposed model, the calculations for RBEC-M benchmark problem published by the International Atomic Energy Agency and derived from the 900MWt RBEC-M reactor design is performed. Geometry of the calculation model of the RBEC-M reactor is shown in Figure 2. The homogenized model includes 12 physical zones, differing from each other by volume fractions and temperatures of materials. Mixed uranium-plutonium nitride fuel is used in the three core zones, which is composed of reactor-grade plutonium recovered from typical light water reactor spent fuel and depleted uranium with $0.1 \mathrm{wt} . \%$ of ${ }^{235} \mathrm{U}$. The core zones are surrounded by lateral (radial) blankets. The structural material is stainless steel and the coolant material is lead-bismuth eutectic. The temperatures of elements in core zones and nuclide compositions of fresh fuel and other materials in 12 physical zones are given in Ref.[9]. The 230-group ACE-formatted data of homogenized mixtures in 12 physical zones are produce by MGGC and MGXSMC based on 230-group cross section library in MATXS format generated by NJOY.

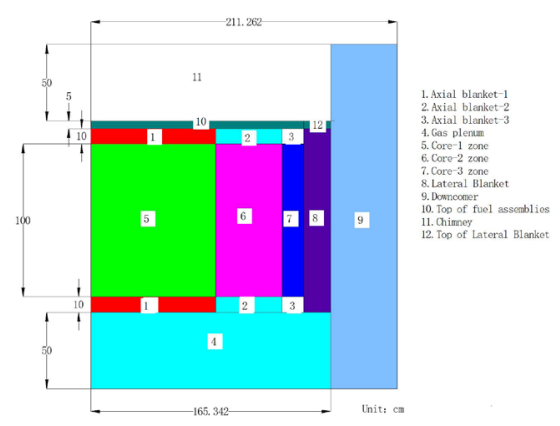

Figure 2. Geometry model of the RBEC-M reactor

\section{Results Analysis}

\subsection{Comparison of scattering angular PDF}

The comparison of scattering angular PDF of the mixture in core-1 zone between truncated Legendre series representation and exponential representation obtained by the maximum entropy method is performed. As shown in Figure 3, Figure 4 and Figure 5, probability density functions of the mixture in core- 1 zone for neutron scattering from group 88, 90 and 89 to group 116,112 and 89 respectively with $\mathrm{P}_{5}$ scattering cross sections are chosen according to the degree of neutron scattering anisotropy. As shown in Figure 3, the anisotropic scattering is not strong and there is only one peak without negative values in the entire angular region. The angular curve of exponential form is

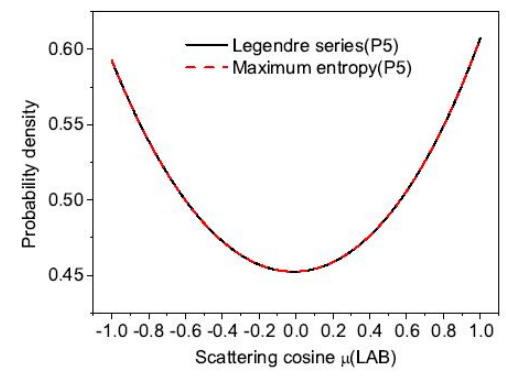

Figure 3. Exponential representation compared to the Legendre series truncated at order 5 for neutron scattering from group 88 to group 116

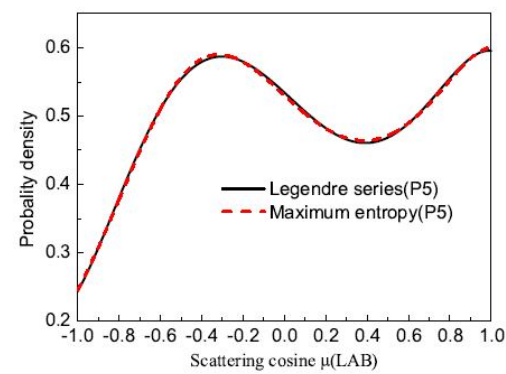

Figure 4. Exponential representation compared to the Legendre series truncated at order 5 for neutron scattering from group 90 to group 112

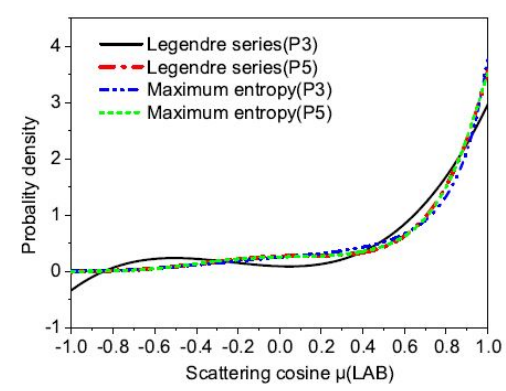

Figure 5. Exponential representation compared to the Legendre series truncated at order 3 and 5 for neutron scattering from group 89 to group 89

in consistent with the curve of the truncated Legendre series representation as well. Figure 4 shows the strongly anisotropic scattering case. There are some peaks in the angular PDF curve with positive value in the entire angular region. The angular curve of exponential form is also in agreement with the curve of the truncated Legendre series representation as well. The angular PDF of truncated Legendre series representation with negative values is shown in Figure 5. The anisotropic scattering is strong and there is a sharply peak. The angular PDF constructed by maximum entropy method is positive everywhere and the $\mathrm{P}_{3}$ approximation of exponential representation is in good agreement with the $\mathrm{P}_{5}$ approximations of Legendre series representation and exponential representation. 
Table 1. $k_{k e f f}$ results of different conditions

\begin{tabular}{ll}
\hline Code & $k_{\text {eff }}$ Value \\
\hline DIF3D & 0.99715 \\
TWODANT & 0.99851 \\
MCNP4C(.50c) & 0.99761 \\
RMC(continuous energy) & 0.99867 \\
RMC(P0) & 1.01931 \\
RMC(P5,discrete cosines) & 0.99891 \\
RMC(P5,equi-probable cosine bins) & 0.99876 \\
\hline
\end{tabular}

\subsection{The verification of critical benchmark}

The criticality calculations of RBEC-M benchmark at the beginning of life have been performed by RMC with continuous energy cross sections and 230-group cross sections of the homogenized mixtures in 12 physical zones. Table 1 compares the effective multiplication factors. Results predicted with DIF3D, TWODANT and MCNP4 are given in Ref.[9]. The calculations with deterministic transport cods were performed with 33-group cross sections generated by MC2-2 code based on the ENDF/B-V.2. And the MCNP calculation was performed with continuous energy cross sections based on the ENDF/B-V. Monte Carlo multigroup calculations were carried out by RMC using 230-group cross sections with isotropic scattering cross sections and with anisotropic scattering cross sections available up to the P5 order produced by the discrete angle method and maximum entropy method.

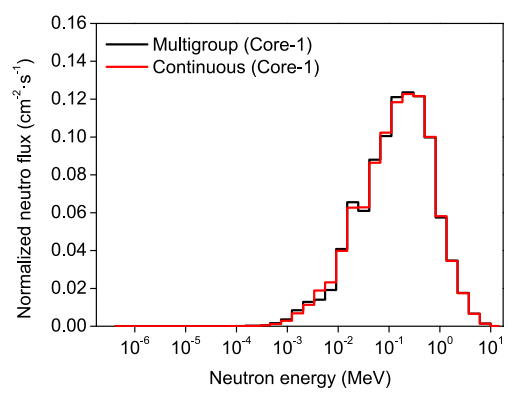

Figure 6. Neutron flux vs energy of core 1

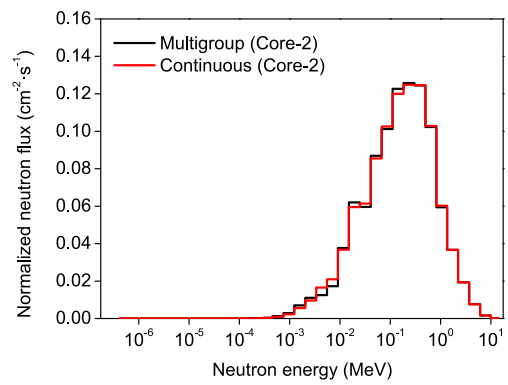

Figure 7. Neutron flux vs energy of core 2

The RMC solutions except for the isotropic $\left(\mathrm{P}_{0}\right)$ scattering case are in good agreement with reference results, indicating angular anisotropy scattering cannot be neglected in criticality calculation of RBEC-M benchmark. It also illustrates that ACE-formatted multigroup cross section library including the neutron scattering angle data computed by the maximum entropy method is credible. Figure 6 and Figure 7 show the comparison of energy spectra between the continuous energy calculation and the multigroup calculation with anisotropic scattering data generated by maximum entropy method in core- 1 and core-2 zones respectively. Results of multigroup calculations are good agreement with continuous calculations. It illustrates that the scattering angle data computed by the maximum entropy method is credible.

\section{Conclusion}

In summary, the secondary neutron scattering angular distribution data of mixtures suitable to multigroup Monte Carlo codes computed by the maximum entropy method can be performed by MGXSMC code. Compared with the truncated Legendre series representation with negative values, the angular scattering distribution of exponential form without negative values is in consistent with the higher order Legendre series representation. The results of criticality calculations show that multigroup cross section library in ACE format including the scattering angle data computed by the maximum entropy method is credible.

\section{References}

[1] X-5 Monte Carlo team, MCNP-A General Monte Carlo Code for N-Particle Transport Code, version 5 manual, LA-UR-03-1987 (Los Alamos National Lab, New Mexico, 2003)

[2] M. B. Emmett, MORSE-CGA: A Monte Carlo radiation transport code with array geometry capability, ORNL-6174 (Oak Ridge National Lab, TN(USA), 1985)

[3] L. L. Carter, C. A. Forest, Nuclear science and Engineering 59(1), 27-45 (1976)

[4] Li Mao, J. P. Both, J. C. Nimal, Nuclear science and engineering 130(2), 226-238 (1998)

[5] Li Mao, J. C. Nimal, Nuclear science and engineering 136(3), 409-414 (2000)

[6] K. Wang, Z. Li, D. She, et al., Annals of Nuclear Energy 82, 121-129 (2014)

[7] O. B. Moskalev, Transport Theory and Statistical Physics 22(2-3), 347-358 (1993)

[8] R. Qiu, X. Ma, Q. Xu, et al., Proceedings of the 2017 25th International Conference on Nuclear Engineering Vol 3 (American Society of Mechanical Engineers, New York, 2017) V003T02A015

[9] J. J. Sienicki, A. Moisseytsev, W. S. Yang, et al., Status report on the small secure transportable auto-nomous reactor(SSTAR)/lead-cooled fast reactor (LFR) and supporting research and development, ANL-GenIV-089 (Argonne National Laboratory, Chicago, 2008) 